\title{
SIMPLIFIED CONSERVATIVE TESTING METHOD OF TOUCH AND STEP VOLTAGES BY MULTIPLE AUXILIARY ELECTRODES AT REDUCED DISTANCE
}

\author{
Giuseppe Parise, L.F. IEEE, Luigi Martirano, S.M. IEEE, Luigi Parise, M. IEEE, \\ Salvatore Celozzi S.M. IEEE, Rodolfo Araneo, S.M. IEEE \\ DIAEE - Electrical Engineering Division \\ University of Rome "La Sapienza"
}

\begin{abstract}
Grounding Systems (GSs) must be tested periodically in order to maintain the touch voltage and step voltage below a safe value in all the zones of the installation. Measurement of the ground resistance and of the touch and step voltages are typically done by the fall-of-potential method, locating the auxiliary current electrode at remote distance to test the effective behavior of the GS. In urban areas, it could be very complicated or impossible to install the auxiliary current electrode as required, not having area around with sufficient accessibility. At this aim, the paper describes a methodology of using multiple current electrodes at short distances modifying the classic fall-of-potential practice, so that the measurements of touch voltage and step voltage result always conservative. The adequacy of a GS is verified if the values of touch and step voltages, tested inside and in the vicinity of the GS are below the permissible limits, regardless if they are true or conservatively increased. Thus, the measured touch and step voltages by the suggested method, always conservative, allow verifying the adequacy of GSs, in the cases where it is impossible to locate the remote auxiliary electrode.
\end{abstract}

Keywords: grounding systems, grounding tests, measurements of touch and step voltages, substation-grounding systems, adequacy of grounding systems

\section{I - INTRODUCTION}

A safe grounding design has the main objective to provide means to carry currents into the ground under normal and fault conditions without exceeding any operating and equipment limits or adversely affecting continuity of service [1]. During its operation the grounding system (GS) has to assure that a person in the vicinity of grounded facilities is not exposed to the danger of critical electric shock. It is well known that under normal conditions, grounded electrical equipment operates at near zero ground potential. During a ground fault event, the flow of current into the ground causes potential gradients within and around the zone of influence of the GS. Protection against electric shock requires GSs must guarantee to keep touch voltage (TV) and step voltage (SV) to a safe permissible value.

The ground potential rise (GPR) is defined as the maximum electrical potential $U_{E}$ that a GS may attain relative to a remote earth electrode. This voltage, GPR, is equal to the maximum grid current times the grid resistance [1].

The TV is the potential difference $U_{t}$ between the GPR of a grounding grid or system and the surface potential where a person could be standing while at the same time having a hand in contact with a grounded structure or object. Figure 1 shows the ground potential profile during a ground fault: $U_{G}$, is the maximum electrical potential that the GS might attain relative to a distant grounding point assumed to be at the potential of remote earth [2]. The GPR is equal to the product between the current to ground $\mathrm{I}_{\mathrm{G}}$, part of the ground fault current $\mathrm{I}_{\mathrm{F}}$, and the ground resistance $\mathrm{R}_{\mathrm{G}}$ (or impedance
$\mathrm{Z}_{\mathrm{G}}$ ) of the ground grid $\mathrm{G}$. In the following, $\mathrm{I}_{\mathrm{G}}$ is assumed equal to $\mathrm{I}_{\mathrm{F}}$.

The SV is the difference in surface potential $U_{s}$ that could be experienced by a person bridging a distance of $1 \mathrm{~m}$ with the feet without contacting any grounded object [2].

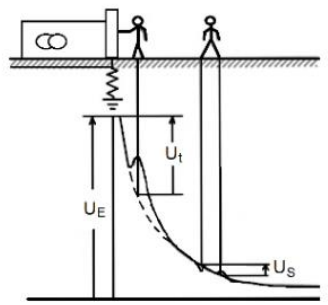

Figure 1. The ground potential rise (GPR) $U_{E}$, the touch voltage $U_{t}$ and the step voltage $\mathrm{U}_{\mathrm{s}}$.

\section{II - TESTING BY THE FALL-OF-POTENTIAL METHOD}

An adequate GS provides a low resistance to remote earth in order to minimize the GPR.

In power installations exceeding $1 \mathrm{kV}$ a.c., the adequacy of a GS is verified if the GPR is below the tolerable TV (or two times its value), for an admitted duration (see Table 5 and $\S 10.3 .1$ of Standard IEC 61936 [3]). In the practice, the GS adequacy can be verified by the measurement of the TVs and the SVs, if the GPR exceeds the safety admissible values. If their values, in the various locations, don't exceed admissible limits, the GS is certainly adequate apart from the value of GPR. There are several methods for measuring GPR of GSs. Among them, the fall-of-potential (FoP) method is most widely applied for almost all types GSs, as proven in many field tests [1-4]. All measurements can be performed with the GS in its normal operative configuration, with all external connections kept in place.

The FoP method consists in applying a voltage between the GS under testing (Electrode G) and a remote auxiliary current electrode $A$ that causes the circulation of a current $I_{F}$ through it (Figure 2). An auxiliary potential probe $\mathrm{P}$ is placed at various positions between A and the GS boundary. The effective difficulty involves the acceptable location of A that has to be located outside the zone of influence of the GS. The position of $\mathrm{P}$ with regard to A may differ.

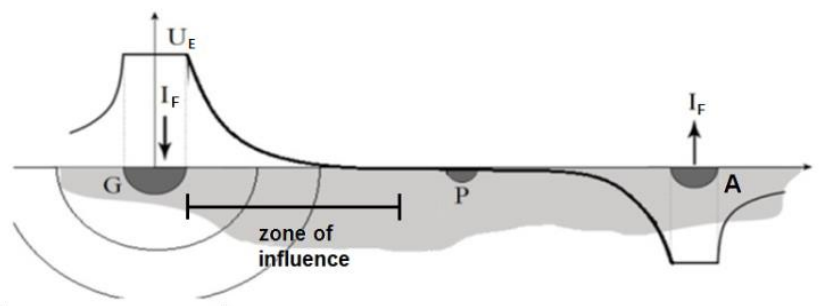

Figure 2. Potential profile between the ground electrode $\mathrm{G}$ and the auxiliary current electrode A. 


\section{CONSERVATIVE TOUCH POTENTIAL MEASUREMENTS BY MULTIPLE AUXILIARY ELECTRODES AT REDUCED DISTANCE}

In the cases where the area of measurements has a reduced accessibility that is also without one direction free of interference, the paper suggests a conservative testing method. The goal of the method is to offer a practical way to verify the adequacy of GSs. In other papers the authors demonstrated that the measurements of TVs and SVs can be done with multiple auxiliary electrodes located at a short distance, since the resulting error is positive and so the results are conservative [5].

In case of a ground fault, a current $\mathrm{I}_{\mathrm{F}}$ circulates in the GS.

This current determines surface potentials in a zone around the electrode defined "influence zone" of the GS (Figure 3).

For the analytic approach, the paper considers a hemispherical testing electrode $\mathrm{G}$, having a radius equal to $\mathrm{r}$, placed in a soil, having a homogenous resistivity $\rho$. These conditions simplify the approach and allow defining general rules of the behavior of GSs.

For the real cases where the soil is characterized by a nonhomogenous resistivity and with the interference of unknown other grounding systems, it is not easy to furnish analytic expressions. In these cases it is fundamental to do experimental measurements and to use simulation software programs.

Being $r$ the radius of the electrode under test, the potential profile $U(p)$ referred to a remote point, in each point with a distance $\mathrm{p}=\mathrm{r}+\mathrm{x}$ from the center of the electrode, is equal to:

$$
U(p)=\frac{\rho I_{F}}{2 \pi \cdot p}
$$

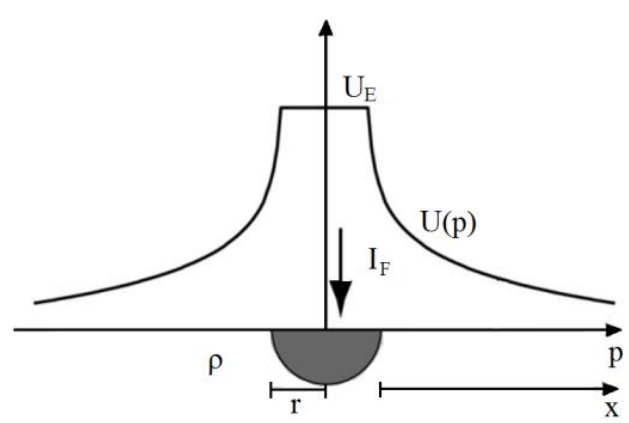

Figure 3. Potential profile $U(p)$ at ground surface, considering a hemispherical GS, with the auxiliary electrode positioned at a distant point and considering an homogeneous soil.

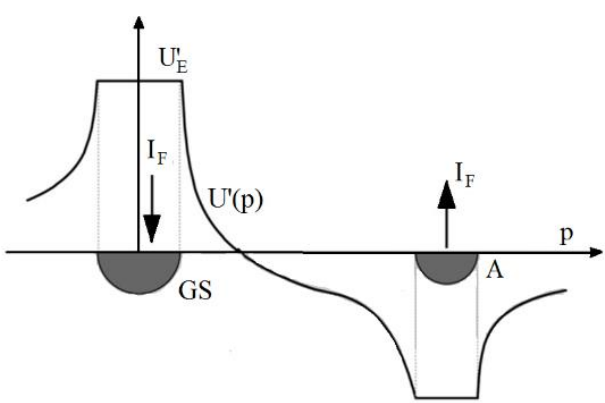

Figure 4. Potential profile $U^{\prime}(p)$ on the homogeneous ground surface with a close auxiliary electrode .

The GPR $\left(\mathrm{U}_{\mathrm{E}}\right)$, assumed by the GS referred to A located at a remote distance, is equal to:

$$
\mathrm{U}_{\mathrm{E}}=\mathrm{R}_{\mathrm{E}} \cdot \mathrm{I}_{\mathrm{F}}
$$

Placing A at a short distance, the potential profile changes as shown in Figure 4.

The GPR (U'E) assumed by the GS under test, referred to A located at a distance $\mathrm{d}$, is equal to:

$$
U_{E}^{\prime}=\frac{\rho I_{F}}{2 \pi \cdot r}-\frac{\rho I_{F}}{2 \pi(r+d)}=U_{E} \cdot\left(1-\frac{r}{r+d}\right)
$$

The relative error due to the proximity of A to GS is equal to:

$$
\mathrm{e}=\frac{U^{\prime} E_{E}-U_{E}}{U_{E}}=-\frac{r}{r+d}
$$

Let's note that e is negative, for example e $=-25 \%$, if $d=3 r$. Figure 5 highlights that when $\mathrm{A}$ is located at short distance, the behavior of the fault current flow produces two diverse distortions:

- A cut effect on the measured value U' ${ }_{\mathrm{E}}$ compared to the true value $\mathrm{U}_{\mathrm{E}}$ so $\mathrm{U}_{\mathrm{E}}{ }^{\prime}<\mathrm{U}_{\mathrm{E}}$;

- A gradient effect on $U_{t}{ }_{t}$ that determines higher values or lower values than the true ones $\mathrm{U}_{\mathrm{t}}$.

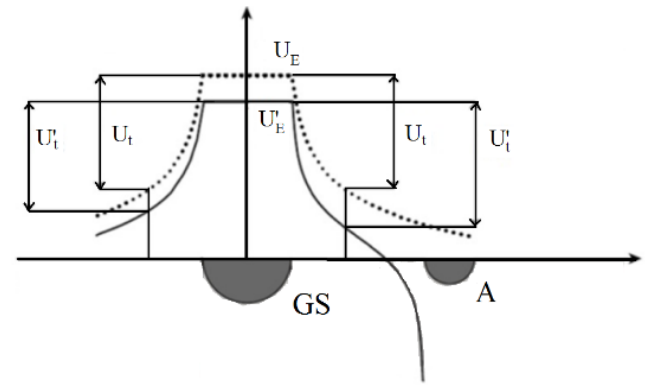

Figure 5. Potential behaviors of a GS (hemispherical) with A at remote point (dashed line) and at short distance (continuous line).

The cut effect depends on the reduction of the ground volume traversed by the current flowing between the GS and A located at shorter distance.

The gradient effect of a single electrode produces an increased flow of current in the region of soil located on the same side of the GS as the current electrode (conservative measurements) and a more reduced flow in the opposite side (not conservative measurements).

Figure 5 shows the generic behavior considering a theoretic case of homogenous soil. This behavior is confirmed by experimental results also in the real cases with nonhomogenous soil (grounding systems in multi-layer soil).

The voltage between the GS and a generic point on the ground surface at distance $p$ from the center of GS, if referred to a remote ground point, is equal to:

$$
U_{E P}=U_{E}-U(p)
$$

Considering $\mathrm{A}$ at a short distance $\mathrm{d}$ the voltage is equal to:

$$
U_{E P}^{\prime}=U_{E}^{\prime}-U^{\prime}(p)
$$

The error $\varepsilon_{\mathrm{UEP}}$ in p.u. is equal to:

$$
\varepsilon_{U E P}=\frac{\left(U_{E P}^{\prime}-U_{E P}\right)}{U_{E P}}=\frac{\left(U_{E}^{\prime}-U_{E}\right)+\left(U^{\prime}(p)-U(p)\right)}{U_{E P}}
$$

The number of the auxiliary electrodes, its distance $d$ and the potential electrode location affect the value of $\varepsilon_{\text {UEPP }}$.

A simple case study is simulated with a GS consisting of a 3 meters long buried rod with a $35 \mathrm{~mm}^{2}$ cross section and 
installing $n$ current auxiliary electrodes $A_{1}, A_{2}, . . A_{n}$. They are rod shaped, 1 meter long, with a cross section of $35 \mathrm{~mm}^{2}$, and are placed around, symmetrically. The injected current is assumed 1 A. Figure 6 shows the relative error $\mathrm{e}_{\mathrm{UEP}}$ computed along a circle of radius equal to $\mathrm{p}=1 \mathrm{~m}$, and considering $\mathrm{d}=2 \mathrm{~m}$, for various number of auxiliary electrodes.

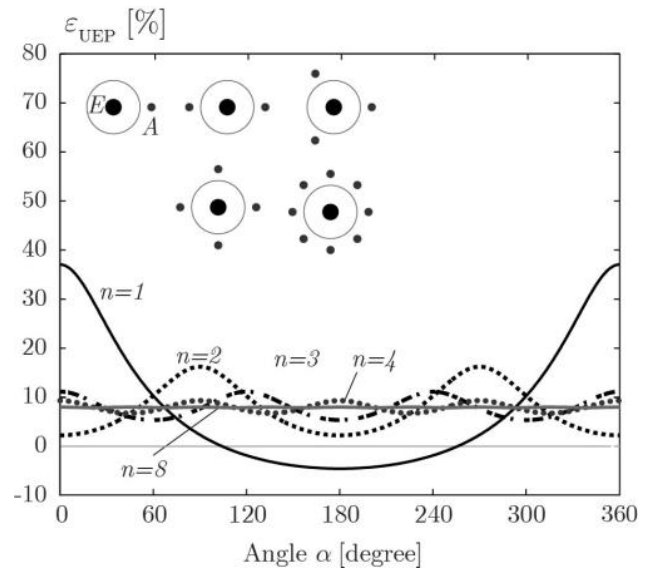

Figure 6. Relative errors as functions of the number of auxiliary electrodes $n$ assuming $p=1 \mathrm{~m}$ and $d=2 m$.

It is possible to observe that:

- by adopting $\mathrm{n}=1$ auxiliary electrode $\mathrm{A}$, the error given by (6) assumes the highest values, but is only conservative in a restricted zone in front of $\mathrm{A}$, where the testing current flows with an increased density than the remaining area around the GS. This behavior guarantees conservative tests for extended GSs with a major penetration in internal parts in front of electrode $\mathrm{A}$.

- by adopting $n \geq 2$ current auxiliary electrodes, the error given by (7) is always conservative because the testing current flows essentially on the superficial layer of the soil around the GS in all the angle values.

- the mean value of the relative error $\mathrm{e}_{\mathrm{UEP}}$ decreases with the increase of the distance toward near zero, at distance out of the zone of the influence ;

- the fluctuations on the mean value of the relative error $\mathrm{e}_{\mathrm{UEP}}$ reduce with the increase of the $n$.

The installation of auxiliary electrodes at short distance and their connections can permanently enable the control of the effectiveness level of GSs by monitoring the touch voltage of one or more pieces of equipment assumed as critical reference. To verify and calibrate the system, initial and periodic measurements can be performed, by means of the classic method with A located at a remote point, when possible.

\section{PRACTICAL GROUNDING MEASUREMENTS METHOD}

The Fall-of-Potential FoP method is the fundamental method for measuring the ground impedance of large GSs [1].

As already mentioned, the FoP method requires circulating a test current $I_{F}$ between the electrode G (the GS under test) and a remote current electrode A (Figure 7).

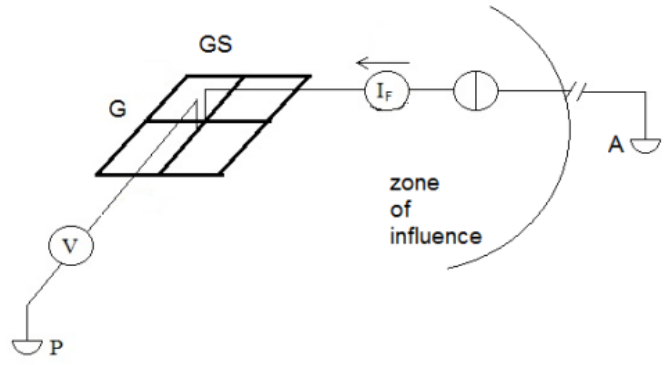

Figure 7. FoP method. A test current $I_{F}$ is circulated between the GS and A. The voltage $\mathrm{V}$ between the GS and $\mathrm{P}$ is measured.

The accuracy of GS resistance measurements depends on the location of A. As demonstrated above, the adoption of multiple auxiliary electrodes, symmetrically installed around the GS, offers a practical conservative measurement method because the electrodes can be located close to the GS. In IEC Standard [3], the minimum distance d from the boundary of the GS and $\mathrm{A}$ is assumed equal at least 4 times the diameter D of the equivalent circumscribed circle of the GS [4].

Measurements of a GS with low impedance require high values of testing currents raising the questions of safety for measurements personnel, the public, and domestic animals.

Moreover, multiple auxiliary electrodes help to ensure greater safety conditions in the execution of the test, since it shares the test current on multiple electrodes, reducing the potential that would set globally on the single remote electrode system.

In order to validate the method and the mentioned effects, a case study of an HV/MV substation GS is studied by a simulation program [7-11].

The GS consists in a typical grounding grid $60 \times 40 \mathrm{~m}$ (with a $\mathrm{D}=72 \mathrm{~m}$ ), with a mesh size of $5 \mathrm{~m}$ (Figure 8 ). A current of 1 A is used. A soil resistivity of $100 \mathrm{ohm} \cdot \mathrm{m}$ is assumed.

Auxiliary electrodes located along the $x$ and $y$ axis In a first simulation the testing method adopted consists in 4 auxiliary electrodes located symmetrically along the $\mathrm{x}$ and $\mathrm{y}$ axis, at distances of $36 \mathrm{~m}$ (0.5D), 72m (D) and 144m (2D) as shown in Figure 8.

The results shown in Figures 9 and 10 confirm that the use of multiple symmetrical current electrodes at short distance guarantees conservative measurements and reduces the errors in all the peripheral zone, outside-inside, around the grounding system in the zones in front of the electrodes, due to the sharing of the test current among more electrodes (conservative behavior).

Figure 11 shows a test simulation done with only one auxiliary electrode located along the positive $\mathrm{x}$ axis. The results confirm that a single auxiliary electrode is useful to analyze the GS internal touch voltages.

Auxiliary electrodes located along the diagonals

A second simulation is done with 4 auxiliary electrodes located symmetrically along the diagonal directions (Figure 12). The results shown in Figures 13 and 14 confirm the conservative behavior of the testing methodology suggested. 


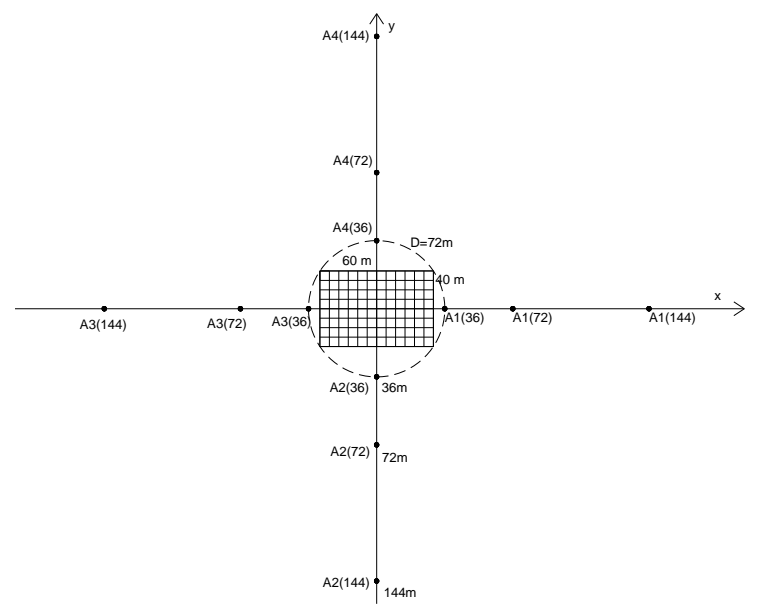

Figure 8 . Case study with the auxiliary electrodes located along the $\mathrm{x}$ and $\mathrm{y}$ axis.

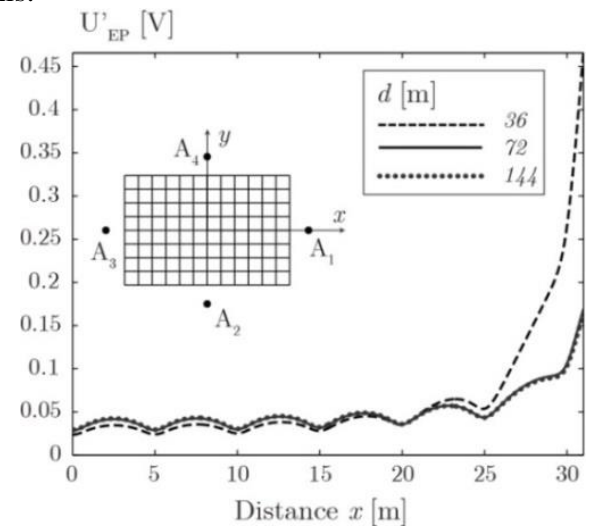

Figure 9. U' ${ }_{\mathrm{EP}}$ profiles adopting 4 electrodes at distances of $\mathrm{d}=36$, $72,144 \mathrm{~m}$ along the $\mathrm{x}$ axis.

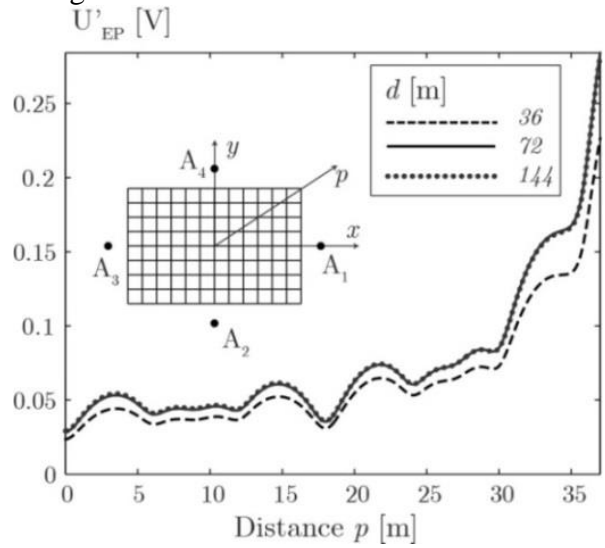

Figure 10. U' ${ }_{E P}$ profiles adopting 4 electrodes at distances of $\mathrm{d}=36$, $72,144 \mathrm{~m}$, along the diagonal.

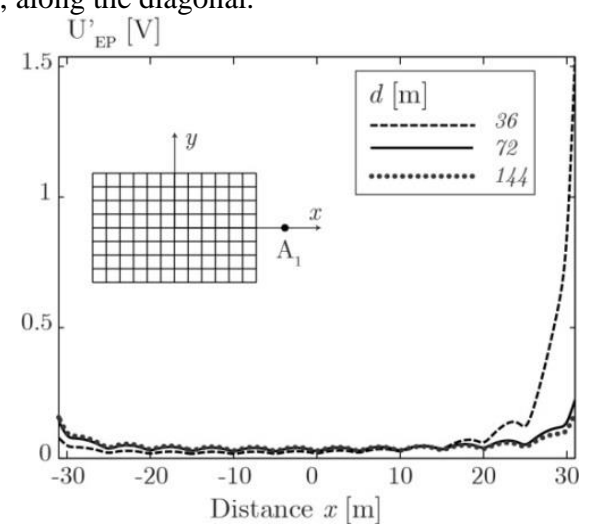

Figure 11. U' electrode at the distance $\mathrm{d}=36,72,144 \mathrm{~m}$.

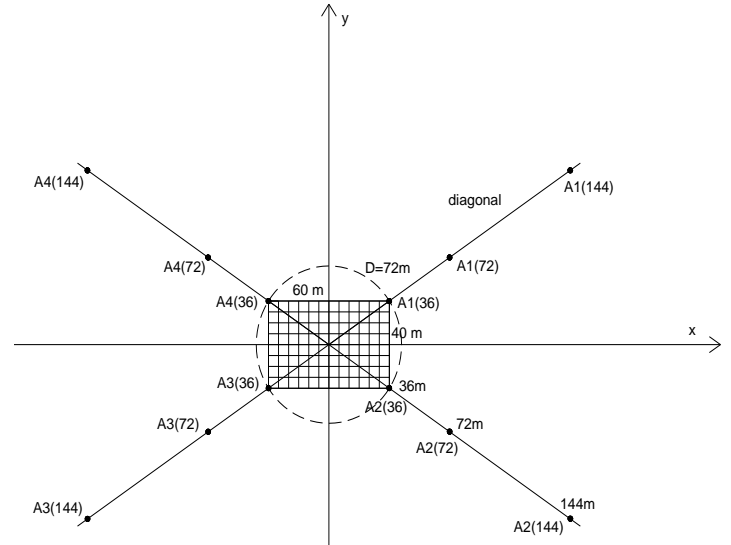

Figure 12. Case study with the auxiliary electrodes located along the diagonals.

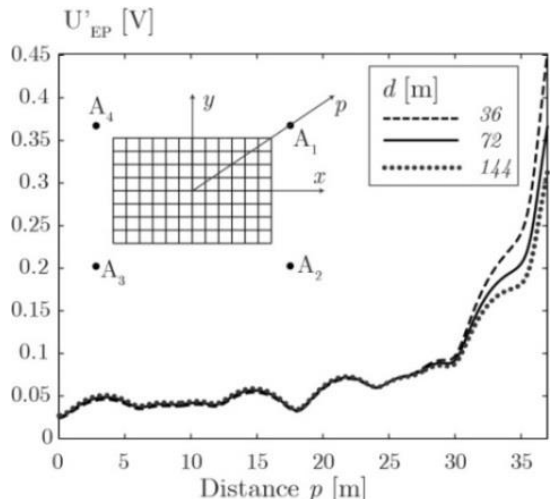

Figure 13. U'EP profiles adopting 4 electrodes at distances of $d=36$, $72,144 \mathrm{~m}$, along the diagonal.

$$
\mathrm{U}_{\mathrm{EP}}[\mathrm{V}]
$$

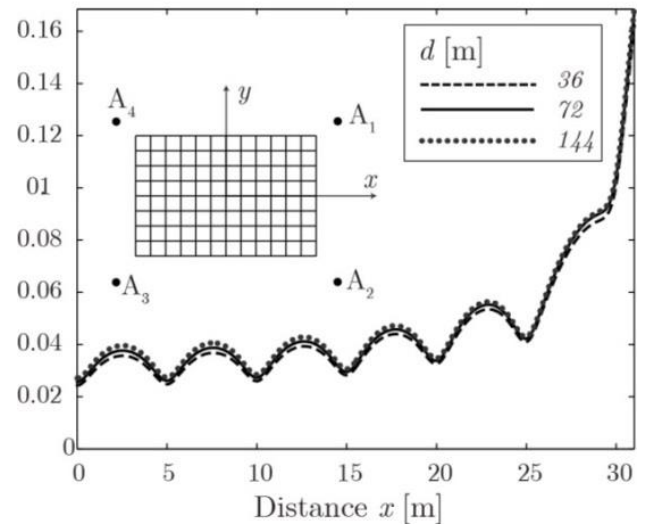

Figure 14. U' ${ }_{\text {EP }}$ profiles adopting 4 electrodes at distances of $\mathrm{d}=36$, $72,144 \mathrm{~m}$ along the $\mathrm{x}$ axis.

$$
\mathrm{U}_{\mathrm{EP}}^{\prime}[\mathrm{V}]
$$

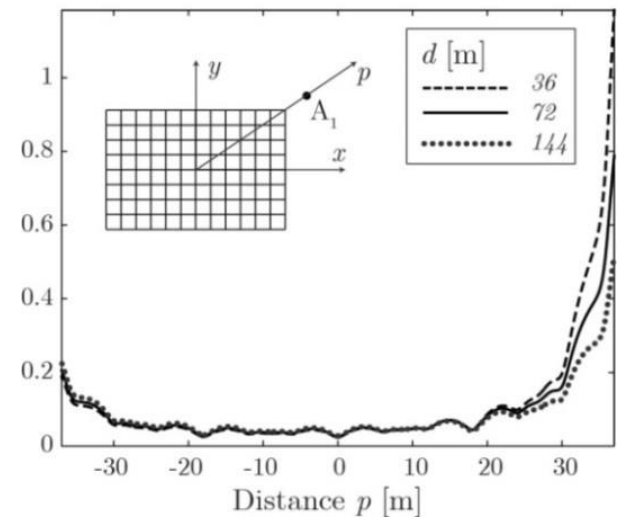

Figure 15. U' ${ }_{\mathrm{EP}}$ profiles adopting only one electrode at distances of $\mathrm{d}=36,72,144 \mathrm{~m}$ along the diagonal. 
Practical methodology by using multiple current electrodes located at short distance.

The results of the simulations confirm the conservative behavior and the methodology of locating multiple current electrodes at short distances modifying the classic FoP practice.

It remains defined a practical procedure with general rules so that the values of touch voltage and step voltage result always conservative (Figure 16) in internal zones and in peripheral zones of an extended symmetrical GS.

Internal zones. The use of only one auxiliary electrode at short distance permits to evaluate the touch and step voltage in internal zones in front of the auxiliary electrode with conservative results. The operator logs on a table the measured values with only one electrode, for the points previously measured in the internal zone in proximity of the auxiliary electrode.

Peripheral zones. The use of multiple symmetrical current electrodes (i.e. 4) at short distance reduces the errors in all the peripheral zones, outside-inside, around the GS.

A good practice is to place the current electrodes symmetrically around the GS, in subsequent steps rotating their position:

- centrally located to the sides, locations A in Figure 16,

- located along the diagonals, in front of the edges, locations B in Figure 16.

The operator logs on a table the measured values, for all the potential points, assuming for each ones the highest measured value.

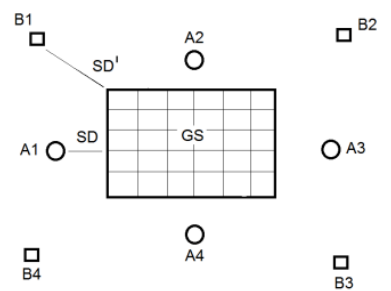

Figure 16. Example of a practical procedure. 4 electrodes are located in a first placement in the locations A1, A2, A3, A4 and in a second placement in the locations B1, B2, B3, B4.

For asymmetrical GS, the use of a simulation program helps to identify the electrodes location to obtain the best results.

\section{V- CONCLUSIONS}

The adequacy of GSs has to be verified periodically in the operational time. In the urban or industrial areas, it is very rare to have around areas with sufficient accessibility to choose suitable locations for auxiliary electrodes and so rigorous ground resistance measures can result impossible. This paper has suggested practical methodologies for testing touch voltage and step voltages that allow to verify the GSs adequacy in areas with reduced accessibility and to monitor its evolution in the time.

\section{REFERENCES}

[1]. IEEE Guide for Safety in AC Substation Grounding, IEEE Standard 80, 2000.

[2]. IEEE Std 81 2012- Guide for measuring earth resistivity, ground impedance and earth surface potential of a grounding system.

[3]. IEC 61936-1 Power installations exceeding $1 \mathrm{kV}$ a.c. Part 1 : Common rules 2010-11
[4].EN 50522: 2010-11, "Earthing of Power Installations Exceeding $1 \mathrm{kV}$ a.c.

[5]. G. Parise, M. Lucheroni, "Measurements of Touch and Step Voltages Adopting Current Auxiliary Electrodes at Reduced Distance" Transactions IEEE/IAS volume 44 n.6 November December 2008

[6]. G. Zizzo, A. Campoccia, E. Riva Sanseverino: “A new model for a safety analysis in a system of earthing systems interconnected through bare-buried conductors", COMPEL Vol. 28 n.2, pp. 412-436.

[7]. Z. Li and J. Fan, "Numerical calculation of grounding system in low frequency domain based on the boundary element method," Int. J. Numer. Methods Engrg., vol. 73, pp. 685-705, May 2008.

[8]. Z. Li, J. Fan, and W. Chen, "Numerical simulation of substation grounding grids buried in both horizontal and vertical multilayer earth model," Int. J. Numer. Methods Engrg., vol. 69, pp. 2359-2380, Aug. 2007.

[9].Z.-X. Li and W.-J. Chen, "Numerical simulation grounding system buried within horizontal multilayer earth in frequency domain,” Int. J. Numer. Methods Engrg., vol. 23, pp. 11-27, Jun. 2007.

[10]. Z. Li, W. Chen, J. Fan, and J. Lu, "A novel mathematical modeling of grounding system buried in multilayer earth," IEEE Trans. Power Delivery, vol. 21, no. 3, pp. 1267-1272, Jul. 2006.

[11]. A. F. Otero, J. Cidras, and J. L. Alamo, "Frequencydependent grounding system calculation by means of a conventional nodal analysis technique," IEEE Trans. Power Delivery, vol. 14, no. 3, pp. 873-878, Jul. 1999.

\section{BIOGRAPHIES}

Giuseppe Parise (M'82-SM'03-F'10-LF'15)) received his M.S degree in Electrical Engineering from the Sapienza University, Rome Italy in 1972. Since 1973, he has been with the Department of Electrical Engineering, Faculty of Engineering, Sapienza University of Rome, where he is currently a Full Professor of Electrical Power Systems. He has been Designer of power electrical systems in buildings complexes as in: the Sapienza University City, the Engineering Faculty, the Polyclinic Umberto I, the Italian Parliament and the Campus Biomedical Research Center. He has authored about 270 papers and is the holder of two patents and three Prize Paper Awards by IEEE/IAS PSD. Prof. Parise has been a Member of Superior Council of Ministry of Public Works since 1983. He is active in the IEEE Industry Applications Society (past Member at Large of Executive Board) and is past coordinator of the Electrical Engineering Area of Sapienza University, chair of Italy Section Chapter IA34, a Member of subcommittees of the Italian Electrical Commission (CEI) and of the Electrical Italian Association (AEIT), the vice President of Association Safety Technology Research for Industry (ASTRI), a past President of AEIT Rome's Section. Since 1975 he has been a Registered Professional Engineer in Italy.

Luigi Martirano (StM'98-M02-SM11) received the M.S. and $\mathrm{Ph} . \mathrm{D}$. degrees in electrical engineering from the University of Rome "La Sapienza", Rome, in 1998 and 2002, respectively. He is currently a researcher in electrical power systems and assistant professor of Building Automation and Energy Management. At Sapienza University $\mathrm{He}$ has authored more than 80 papers in international journals and conferences and one international patent. His research activities cover power systems design, planning, safety, lightings, home and building automation, energy management. He is a senior member of the IEEE/IAS, member of the AEIT (Italian Association of Electrical and Electronics Engineers) and of the CEI (Italian Electrical Commission) Technical Committees CT205 (Home and Building Electronic 
Systems) and CT315 (Energy Effectiveness). He has been Registered Professional Engineer.

Luigi Parise (StM'06-M'10) received the B.S. degree (three years), the M.S. degree (two years) and Ph.D. degree (three years) in Electrical Engineering from the Sapienza University of Rome, Rome Italy, in 2007, 2009 and 2014 respectively. He is currently Research Fellow at University of Calabria (UNICAL) and he is Tutor in electrical power systems in hospitals in the University Biomedical Campus of Rome.

Mr. Parise was a recipient of the 2010 Italian Electrotechnical Committee (CEI) Award for the Best Thesis titled: Electrical Service Continuity In Critical Buildings - Protection of Hospitals Against Lightning.

$\mathrm{He}$ is a member of the Electrical Italian Association (AEIT), the R8/Europe Area Chair of IEEE Industry Applications Society SBCs and the Secretary of Italy Section IAS Chapter. Since 2008, he has been a Registered Professional Engineer in Italy.

Salvatore Celozzi (M'92-SM'97), Ph. D., is Full Professor of the Electrical Engineering Divison of DIAEE, University of Rome "La Sapienza". Dr. Celozzi published about 130 papers in international journals or conference proceedings, mainly in the fields of electromagnetic shielding and transmission lines. He has won several best paper awards served as Associate Editor of the IEEE Transactions on Electromagnetic Compatibility (1995-2000), and authored a book on Electromagnetic Shielding for Wiley in 2008. He served as Chair of the IEEE EMC Society Chapter of Italy for more than 10 years. His current research interests include transmission lines, electromagnetic compatibility, meta- and piezomaterials, and numerical methods. Since 1990, he is a Registered Professional Engineer in Italy.

Rodolfo Araneo (M'03,SM'10) received the M.S. (cum laude) and $\mathrm{Ph} . \mathrm{D}$. degrees in electrical engineering from the University of Rome "La Sapienza", Rome, in 1999 and 2002, respectively. He has won several awards. Dr. Araneo received the Past President's Memorial Awards in 1999 from IEEE Electromagnetic Compatibility (EMC) Society. His research activity is mainly in the eld of EMC and includes numerical and analytical techniques for modeling high-speed printed circuit boards, shielding, and transmission line analysis. He is Registered Professional Engineer in Italy. He has authored more than 110 papers in international journals and conference proceedings. 